\title{
Cascaded Continuous-Wave Raman Frequency Conversion in External- Cavity Diamond Lasers
}

\author{
Robert J. Williams' ${ }^{1}$, David J. Spence ${ }^{1}$, Oliver Lux ${ }^{2}$, and Richard P. Mildren ${ }^{1}$ \\ ${ }^{1}$ MQ Photonics Research Centre, Department of Physics and Astronomy, Macquarie University, Sydney, NSW 2109, Australia \\ ${ }^{2}$ Deutsches Zentrum für Luft- und Raumfahrt (DLR), Institut für Physik der Atmosphäre, Oberpfaffenhofen 82234, Germany
}

High-brightness continuous-wave (cw) beams at various wavelengths in the near and mid-infrared, visible, and ultraviolet are in demand for numerous applications spanning defence, astronomy, space-science, medicine and remote-sensing. While fiber lasers are an attractive solid-state technology for high-brightness cw beams, their output wavelengths are restricted to $1-1.1 \mu \mathrm{m}$ (for ytterbium), $2 \mu \mathrm{m}$ (thulium) and their harmonics. Nonlinear frequency conversion, in OPOs/OPAs and with Raman conversion, is often used as a means for converting beams to a desired wavelength, but thermal loading in the nonlinear crystal typically leads to beam distortions at higher powers, limiting beam brightness.

Diamond has recently enabled efficient cw frequency conversion from 1.06-1.24 $\mu \mathrm{m}$, at high powers while maintaining diffraction-limited beam quality, using fiber laser and Nd:YAG pumps [1-3]. Diamond's high Raman gain and extreme thermo-mechanical properties have enabled record output powers up to $0.38 \mathrm{~kW}$ [3]. The use of an external-cavity design with non-resonant pumping facilitates conversion of conventional pump sources [4], such as fibers, without requiring ultra-narrow linewidth or cavity-locking/stabilization, and the automatic phasematching of stimulated Raman scattering removes any further angle or temperature stabilization requirements. However, cascaded Stokes shifting in the cw external-cavity regime has only been reported in barium nitrate, and exhibited very limited conversion efficiency $(<0.5 \%)[5]$.

Here we report efficient frequency conversion from 1.06-1.49 $\mu \mathrm{m}$, via the second Stokes shift in diamond with output power in excess of $100 \mathrm{~W}$ and a 55\% linear slope efficiency [6], with immediate potential for extension to $1.56 \mu \mathrm{m}$ using ytterbium fiber pump lasers operating at $1.1 \mu \mathrm{m}$ in order to reach the eye-safe and atmospheric transmission band desired for many space and defence applications. We also report an analytical model for cascaded Raman lasing in an extra-cavity configuration, revealing the design parameters required for efficient conversion on second, third and higher Stokes shifts. The trends predicted by the model in the case of a second Stokes laser are demonstrated in the experimental results, and by varying the cavity parameters we were able to demonstrate both an efficient regime, and an alternate and surprising regime in which Raman power conversion to both first and second Stokes is suppressed in a highly-resonant cavity. The combination of high-brightness fiber lasers and efficient frequency conversion in diamond, now via cascaded Raman shifting, has the potential to provide cw beams at applications-rich wavelengths with much higher brightness than is currently available.
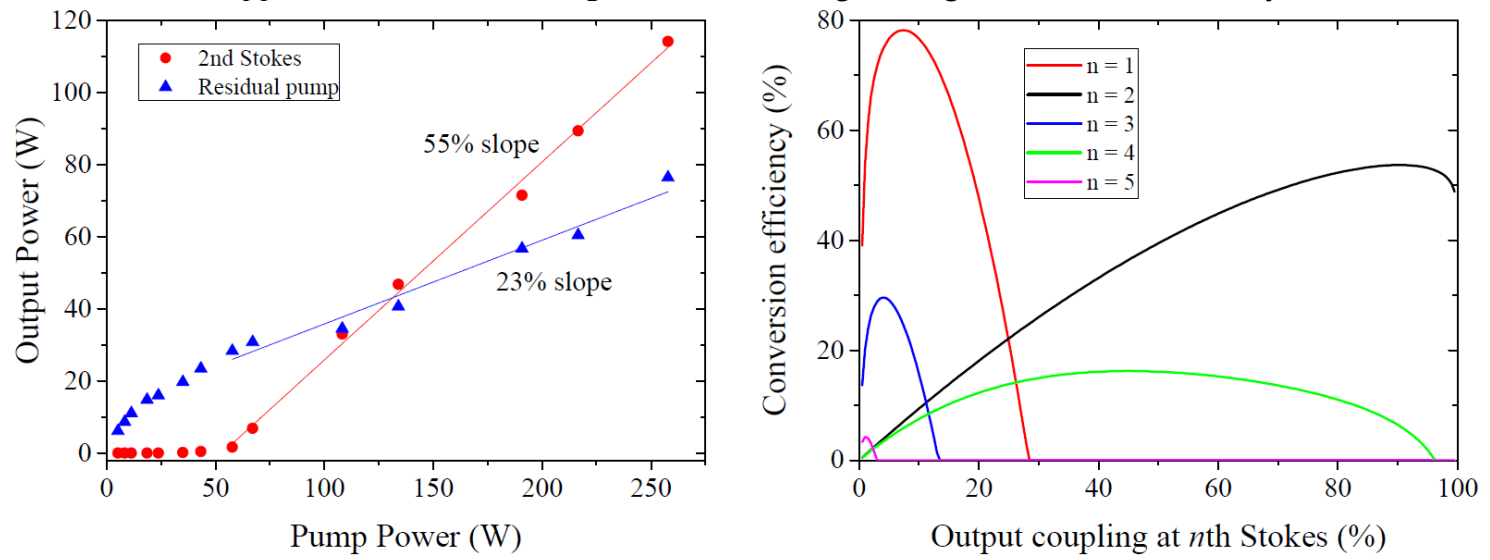

Fig. 1 (a) Output of the second Stokes diamond laser. (b) Predicted conversion efficiency as a function of output coupling for first, second, third and fourth Stokes lasers with $300 \mathrm{~W}$ pumping at $1.06 \mu \mathrm{m}$.

\section{References}

[1] O. Kitzler, A. McKay, and R. P. Mildren, "Continuous-wave wavelength conversion for high-power applications using an external cavity diamond Raman laser," Opt. Lett. 37, 2790-2792 (2012).

[2] R. J. Williams, O. Kitzler, A. McKay, and R. P. Mildren, "Investigating diamond Raman lasers at the $100 \mathrm{~W}$ level using quasicontinuous-wave pumping," Opt. Lett. 39, 4152-4155 (2014).

[3] R. J. Williams, J. Nold, et al., "Efficient Raman frequency conversion of high-power fiber lasers in diamond," Laser Photonics Rev. 9, 405-411 (2015).

[4] A. S. Grabtchikov, V. A. Lisinetskii, et al., "Multimode pumped continuous-wave solid-state Raman laser," Opt. Lett. 29, 2524-2526 (2004).

[5] A. S. Grabtchikov, V. A. Lisinetskii, et al., "Continuous-wave solid-state two-Stokes Raman laser," Quantum Electron. 39, 624 (2009)

[6] R. J. Williams, D. J. Spence, O. Lux, and R. P. Mildren, "High-power continuous-wave Raman frequency conversion from 1.06 um to $1.49 \mu \mathrm{m}$ in diamond," Opt. Express 25, 749-757 (2017). 